\title{
Study on the Control of Porosity in Films of Polythiophene Derivatives
}

\author{
Guillem Ruano, ${ }^{a}$ Carlos Alemán,,${ }^{a,}$ and Juan Torras ${ }^{a, *}$ \\ ${ }^{a}$ Department of Chemical Engineering (EEBE) and Barcelona Research Center for Multiscale \\ Science and Engineering. Universitat Politècnica de Catalunya, C/Eduard Maristany 10-14, Ed \\ I2, 08019, Barcelona, Spain
}

*Corresponding Authors: carlos.aleman@upc.edu and joan.torras@upc.edu 


\begin{abstract}
Conducting polymers typically exhibit different oxidation states, which are easily interchangeable among them by means of the application of an electrical potential. In this work, we present a theoretical and experimental study to regulate the pore size of poly $(3,4-$ ethylenedioxythiophene) (PEDOT) films doped with $\mathrm{ClO}_{4}{ }^{-}$ions by controlling their oxidation state. More specifically, different bulk and surface PEDOT models have been evaluated applying 2D- and 3-D periodic boundary conditions to density functional theory calculations. In highly oxidized PEDOT films, calculations predict that the incorporation of dopant ions increases the separation between neighboring chains, causing a structural re-organization. Thus, the calculated average pore size, which has been modeled as a structural defect in 2D surface models, increases by $15.1 \%$. This increment is consistent with experimental measures of the nanopore size in PEDOT films with enhanced porosity, which reflect a difference of $25.2 \%$ between the oxidized and reduced forms. This superficial phenomenon could easily be used to retain and release controlled drugs through the application of different electric potentials.
\end{abstract}




\section{Introduction}

The ability to create controlled interfaces, such as nonporous and nanoperforated membranes, has been an important topic of study during the last decade, particularly in the biomedical field.[1-4] Among them, polymeric free-standing nanomembranes (from 1 up to $100 \mathrm{~nm}$ thick) have emerged as versatile elements for biomedical applications such as overlapping therapy, burn wound infection treatment, antimicrobial platforms, scaffolds for tissue engineering, drugloading and delivery systems, biosensors, etc.[5-7] Moreover, the utilization of electrically conducting materials and devices for biomedical and biotechnological applications has become an interesting topic to the community due their potential applications.[8-10] In fact, intrinsically conducting polymers (ICPs) are perceived as suitable candidates for these biomedical devices because of both their luminescence properties,[11] electrical[12, 13] behavior and biocompatibility.[14, 15] These interesting properties were previously exploited on the use of ICP as electro-chemo-mechanical actuators that can be envisaged as artificial muscles.[16-21]

Recently, new nanomembranes were created for biomedical and biotechnological applications using ICPs based on poly(thiophene) and its derivatives.[22] Moreover, a novel methodology was developed to create nanoperforated nanomembranes[23] to fix biological material in a welldefined nano-holes, thus, increasing the potential applicability of those nanomembranes.[4] Taking into account the state-of-the-art, an interesting application can be envisaged for nanoporous ICP ultra-thin membranes as a potential drugs releaser by controlling the nanopore size. More specifically, the release of drugs immobilized inside the nanopores could be regulated by changing the nanopore size through the electro-chemo-mechanical properties of ICPs. In some circumstances, ICP films can be considered as motors driven by reversible electrochemical reactions (Faradaic motors).[24, 25] Thus, electrons are extracted from or injected to polymeric 
chains during the reactions generating positive or negative chains, respectively, while hydrated counterions (i.e. anions or cations accompanied with water molecules) are exchanged between the polymeric matrix and the electrolyte to keep the charge balance inside the film (Fig. 1a). Such electronic and ionic charge transport processes cause conformational movements in the polymer chains that, together with the compositional variation inside the polymeric matrix (i.e. entrance and scape of hydrated ions), guarantee film volume variations during reversible oxidation and reduction reactions (swelling and shrinking, respectively). Within this context, it should be mentioned that this electrochemically induced actuation mechanism was recently used to regulate the drug delivery from polyester microfibres loaded with poly $(3,4-$ ethylenedioxythiophene) (PEDOT) nanoparticles, which exhibited a volume variation of $\sim 17 \%$ upon the application of electric pulses, increasing the porosity of the microfibers.[26]

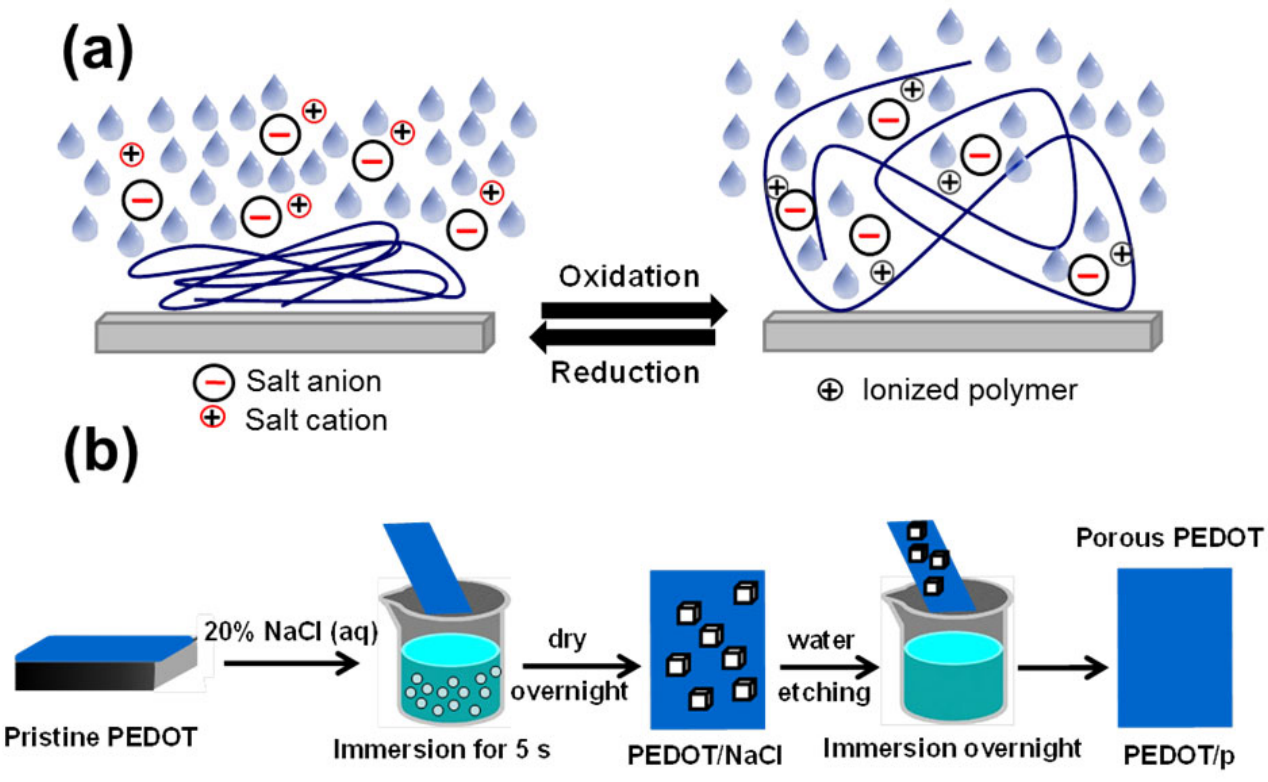

Fig. 1. (a) Reaction induced swelling/shrinking of an ICP film. (b) Preparation of porous PEDOT growing $\mathrm{NaCl}$ crystals on the surface of the film and etching them with water. 
Concepts relating the volume variation of macroscopic ICP films with oxidation and reduction reactions (i.e. with the doping level) can be extrapolated to microscopic nanopores and nanoperforations for regulating the transport of medium size molecules in drug delivery applications. Indeed, chemical and physical properties of ICPs are, in general, intimately related with their doping level.[27] For example, experimental and theoretical studies on oxidized (doped) and reduced (dedoped) PEDOT showed important structural differences (e.g. the interchain distance was shorter for the latter than for the former).[28, 29] Moreover, the cohesion between polymer chains was found to increase when dopant ions are intercalated among them.[30] Within the context of structure-electronic properties relationships, theoretical calculations on ICPs are usually conducted using either oligomers with a growing number of repetitive units or applying periodic boundary conditions (PBC) along the direction of growth of the polymer chain (1D-PBC),[31-34] while more complex solid state calculations with 2D- or 3D-PBC approaches have been scarcely reported.[35, 36] The main goal of this work is to evaluate the influence of the doping level on the pore size of ICP films combining advanced theoretical approaches based on 3D-PBC models and experimental measures.

In this work, we use full 2D- and 3D-PBC density functional theory (DFT) calculations to conduct a differential investigation on the structural and electronic changes induced by the doping level. Furthermore, the influence of the doping level on the micro-pore size of porous anodically polymerized PEDOT has been experimentally characterized by scanning electron microscopy (SEM). Results, which have allowed us to check the viability on the pore-size control, are expected to assist for regulating the release of drugs immobilized inside the pores by controlling their diameter through the doping level. 


\section{Methods}

Materials. 3,4-ethylenedioxythiophene (EDOT) and acetonitrile of analytical reagent grade were purchased from Sigma-Aldrich and used as received, without further purification. Anhydrous $\mathrm{LiClO}_{4}$, analytical reagent grade from Aldrich, was stored in an oven at $70{ }^{\circ} \mathrm{C}$ before use in electrochemical experiments. Milli-Q water grade $(0.055 \mathrm{~S} / \mathrm{cm})$ was used in all synthetic processes.

Synthesis of porous PEDOT films. PEDOT films were prepared by chronoamperometry under a constant potential of $1.40 \mathrm{~V}$ and adjusting the polymerization charge to $0.55 \mathrm{C}$. Electrolytic cells made of three-electrode one-compartment were used for all polymerizations under nitrogen atmosphere $\left(99.995 \%\right.$ in purity) at $25{ }^{\circ} \mathrm{C}$. Stainless steel AISI 316 sheets of $1.0 \times$ $1.5 \mathrm{~cm}^{2}$ were used as working and counter electrodes in combination with a reference electrode of $\mathrm{Ag} \mid \mathrm{AgCl}$ containing a $\mathrm{KBr}$ saturated aqueous solution $\left(\mathrm{E}^{\mathrm{o}}=0.222 \mathrm{~V}\right.$ vs. standard hydrogen electrode at $25^{\circ} \mathrm{C}$ ). To avoid interferences during the electrochemical analyses, before each trial the working and counter electrodes were cleaned with ethanol, after that with acetone, and dried in an air-flow.

The electrolytic cell was filled with $40 \mathrm{~mL}$ of a $10 \mathrm{mM}$ acetonitrile solution of EDOT monomer with $0.1 \mathrm{M} \mathrm{LiClO}_{4}$ as doping electrolyte. The experimental set-up for this anodic polymerization was described in previous work.[37] PEDOT porous film were achieved by the growing and etching stages of $\mathrm{NaCl}$ crystals on the surface of the previously obtained PEDOT film using a simple methodology, which is sketched in Fig. 1b. First, $\mathrm{NaCl}$ crystals were grown by plunging the prepared films in a $20 \% \mathrm{w} / \mathrm{v}$ salt aqueous solution during $5 \mathrm{~s}$ and, subsequently, dried in a desiccator overnight. After this, the porous surface was obtained by removing the 
grown salt crystals from PEDOT/ $\mathrm{NaCl}$ films by plunging in water overnight and drying in a desiccator for at least $24 \mathrm{~h}$. Hereafter, the resulting porous PEDOT films are denoted PEDOT/p.

\section{Chemical characterization of oxidized-reduced PEDOT/p films.}

FTIR spectra of PEDOT/p films were recorded on a FTIR Jasco 4100 spectrophotometer. Attenuated total reflection accessory with a diamond crystal (Specac model MKII Golden Gate Heated Single Reflection Diamond ATR) were used to place the samples. A total of 64 scans were performed between 4000 and $600 \mathrm{~cm}-1$ ( $4 \mathrm{~cm}-1$ of resolution) for each sample.

Raman spectra of PEDOT films were recorded using a Renishaw inVia Qontor confocal Raman microscope with $785 \mathrm{~nm}$ laser excitation and a nominal $300 \mathrm{~mW}$ output power directed through a microscope (specially adapted Leica DM2700 M microscope) to the sample. The scattered light is collected and directed to a spectrometer with a 1200 lines $\cdot \mathrm{mm}-1$ grating. The laser power was adjusted to $1 \%$ of its nominal output power with an exposure time of $10 \mathrm{~s}$. Each spectrum was collected with 3 accumulations.

Surface characterization of oxidized-reduced PEDOT/p films. Two different oxidation states were obtained by applying two different potentials to the porous PEDOT/p film. Specifically, chronoamperometries (CA) were conducted applying a constant potential of +1.1 (oxidation) and -1.1 (reduction) $\mathrm{V}$ for $10 \mathrm{~s}$ to PEDOT/p films to obtain oxidized and reduced porous PEDOT films (hereafter PEDOT $/ \mathrm{p}^{\text {oxd }}$ and PEDOT $/ \mathrm{p}^{\text {red }}$ respectively). These experiments were made in cells of three electrodes under a nitrogen atmosphere at $25^{\circ} \mathrm{C}$.

The doping Level $(d l)$ of oxidized and reduced PEDOT/p layers were determined by cyclic voltammetry $(\mathrm{CV})$ using an aqueous solution of $0.1 \mathrm{M} \mathrm{LiClO}_{4}$. The initial and final potential were $-0.5 \mathrm{~V}$, while the reversal potential was $+1.1 \mathrm{~V}$. The scan rate was set to $100 \mathrm{mV} \mathrm{s}^{-1}$. 
Electrochemical estimation of the doping level (dl) was carried out using the following equation:[38]

$$
d l=\frac{2 Q_{o x}}{Q_{p o l}-Q_{o x}}
$$

where $Q_{o x}$ is the charge associated to the oxidation process derived from the latter $\mathrm{CV}$, and $Q_{p o l}$ is the total charge used for the PEDOT film deposition at the generation time.

Film thickness measurements were carried out using a Dektak 150 stylus profilometer (Vecco, Plainview). Several scratches were intentionally caused on the films to allow the film thickness measurement. Imaging of the film was conducted using the following optimized setting: tip radius $=2.5 \mu \mathrm{m}$, stylus force $=1.5 \mathrm{mg}$ scan length $=3 \mathrm{~mm}$ and a scan resolution of $0.33 \mu \mathrm{m}$. Two different measures were obtained: (a) the vertical distances (l), which is the difference between the polymer and substrate height without any average; (ii) the surface roughness $(\boldsymbol{R} \boldsymbol{a})$ as the arithmetical mean deviation of the assessed profile. The density of both reduced and oxidized polymers was determined by the flotation method from $\mathrm{CCl}_{4}+\mathrm{C}_{2} \mathrm{H}_{5} \mathrm{I}$ mixtures.

The X-ray diffraction (XRD) experiments were performed by using Bruker D8 Advance X-ray diffractometer with a monochromatic $\mathrm{Cu}$ radiation $(\lambda=1.5406 \AA)$. Polymer powders were deposited in a silicon wafer and then were fixed by vacuum grease. Finally the fixed PEDOT powder was used in powder XRD measurement.

Scanning electron microscopy (SEM) studies were performed to examine the surface morphology of the prepared films. Dried samples were placed in a Focused Ion Beam Zeis Neon 40 scanning electron microscope operating at $5 \mathrm{kV}$, equipped with an energy dispersive $\mathrm{X}$-ray (EDX) spectroscopy system. The average pore size of each system was obtained statistically from a set of 200 measures distributed among 20 SEM pictures of a total of 3 different samples each. 
Theoretical calculations. Surface nanopore expansion/constriction associated to the reversible transition between dedoped and doped PEDOT systems have been examined by means of six different models covering the three possible states (i.e. bulk, raw surface and nanopored surface). Firstly, two bulk structures were simulated. These consisted of eight EDOT repeated units distributed in two chains, which grew along the lattice vector $c$ using a 3D-PBC approach. In one of them, four $\mathrm{ClO}_{4}^{-}$anions were introduced in order to simulate the doped bulk at an ideal doping level of 0.5 . Initial coordinates were obtained from a previous crystallographic study using 1D-PBC approach.[30] The other four systems were used to model PEDOT surfaces, which were build starting from the previous optimized bulk system but adding a vacuum region of $45 \AA$ along lattice vector $\boldsymbol{b}$. In this case, two of the models simulated the raw surface while the other two simulated the porous nanomembrane. This was achieved building four different supercells: the two simulating the raw surface were based on two repeating bulk units along $\boldsymbol{c}$ direction (doped and dedoped), and the other two were made of two repeating units along $\boldsymbol{c}$ direction while only one along $\boldsymbol{a}$-direction, thus leaving an empty space replicating the porous surface (doped and dedoped).

All calculations were based on the DFT in the standard Kohn-Sham formalism, as implemented in the SIESTA package[39, 40] with PBC. The generalized gradient approximation (GGA) was used on the calculation of exchange-correlation energy employing the Perdew-Burke-Ernzerhof (PBE) functional.[41] All atoms were represented by the TroullierMartins norm-conserving pseudopotentials[42] and a numerical double- $\zeta$ basis set with polarization function. Initial structures were allowed to relax under PBC by means of conjugate gradient minimization. Thus, the atom coordinates were optimized until the forces acting on each atom were smaller than $0.04 \mathrm{eV} / \AA$, using a mesh cutoff of 350 Ry. Sampling of the irreducible 
Brillouin zone was performed according to the scheme proposed by Monkhorst and Pack[43] with a k-points mesh made of $6 \times 4 \times 4$ and $6 \times 1 \times 4$ for the bulk and surface models, respectively.

\section{Results and Discussion}

Dedoped and doped bulk structures. Fig. 2 compares the structures obtained for the dedoped and $\mathrm{ClO}_{4}{ }^{-}$doped bulk PEDOT models (hereafter named $b$-dedoped and $b$-doped model, respectively) after optimization using the 3D-PBC DFT approach. Since the unit cell angles were freely optimized, all resulting systems present a triclinic symmetry. However, some angles show very close values to higher crystal symmetry. In those cases, the crystal unit cell will be denoted as quasi- the closest symmetry. Table 1 compares the structural parameters for both systems with experimental values. Although the calculated $b$-dedoped model presents a quasi-monoclinic unitary cell $\left(\gamma=81.9^{\circ}\right)$ with $\alpha=89.7^{\circ}$ and $\beta=90.0^{\circ}$, the obtained interlayer and interchain distances can be easily compared with the experimental orthorhombic unitary cell presented by Tran-Van et al.[44] In that work, the two most intense diffraction peaks at 7.87 and $10.52 \AA$ of a dedoped PEDOT film were assigned to the distances of periodic structure along lattice vector $c$ (i.e. polymer repeat distance along the chain) and $\boldsymbol{a}$ (i.e. parallel interchain distance, $\mathrm{d}_{\mathrm{R}}$ ), respectively. Those values are in very good agreement with the theoretical parameters of 7.90 and $10.28 \AA$ obtained for the $b$-dedoped system in this work, respectively (Table 1). Furthermore, the inter-chain stacking distance (minimum distance between two adjacent chain planes of thiophene rings, $\mathrm{d}_{\mathrm{H}}$ ) obtained in this work for the simulated model of dedoped PEDOT was $3.44 \AA$, while the 020 reflection plane $(\boldsymbol{b} / 2)$ was $3.68 \AA$. The latter distance can be associated to the third most intense peak of the X-ray diffractogram of Tran-Van et al.[44] with an angle of $25.9^{\circ}$ and $3.43 \AA$. This value is also pretty similar to the stacking distance reported 
for the tosylated-doped PEDOT in a orthorhombic crystal [28]. Also, the torsion between lattice direction $\boldsymbol{a}$ and the plane of PEDOT chain due to interactions between adjacent PEDOT layers reported in the literature with values of $8.1^{\circ}[29]$ and $10^{\circ},[45]$ are similar to the value calculated for the $b$-dedoped model of $14.7^{\circ}$. As in general the concordance between the calculated and experimental values is very good for the bulk models, the 2D-PBC approach described in the Methods section is expected to represent satisfactorily the PEDOT surface.

Table 1. Calculated Values of the Crystal Structures, Supercell Volume (V), Inter-chain Stacking Distance $\left(\mathrm{d}_{\mathrm{H}}\right)$ and theParallel Interchain Distance $\left(\mathrm{d}_{\mathrm{R}}\right)$ of Dedoped and Doped PEDOT. The meaning of $d_{H}$ and $d_{R}$ is sketched in Fig. 2.

\begin{tabular}{|c|c|c|c|c|c|c|}
\hline & \multirow{2}{*}{$\begin{array}{c}\text { Supercell } \\
(\AA ̊)\end{array}$} & \multicolumn{3}{|c|}{$\mathbf{V}$} & \multirow{2}{*}{$\begin{array}{l}\mathbf{d}_{\mathrm{H}} \\
(\AA)\end{array}$} & \multirow{2}{*}{$\begin{array}{l}\mathbf{d}_{\mathrm{R}} \\
(\AA)\end{array}$} \\
\hline & & Angles ( ${ }^{\circ}$ ) & $\left(\AA^{3}\right)$ & Unit cell $(\AA)$ & & \\
\hline \multicolumn{7}{|c|}{$b$-dedoped } \\
\hline \multirow{3}{*}{ DFT } & $\mathrm{a}=10.28$ & $\alpha=89.7$ & & $a=10.28$ & & \\
\hline & $b=7.37$ & $\beta=90.0$ & 1185.2 & $b=7.37$ & 3.44 & 10.28 \\
\hline & $\mathrm{c}=15.80$ & $\gamma=81.9$ & & $\mathrm{c}=7.90$ & & \\
\hline \multirow{3}{*}{ exp.[44] } & & & & $\mathrm{a}=10.52$ & & \\
\hline & & & & $\mathrm{b}=6.86$ & 3.43 & 10.52 \\
\hline & & & & $c=7.86$ & & \\
\hline \multicolumn{7}{|c|}{$b$-doped } \\
\hline \multirow{3}{*}{ DFT } & $\mathrm{a}=12.64$ & $\alpha=86.3$ & & $\mathrm{a}=12,54$ & & \\
\hline & $b=6.59$ & $\beta=88.3$ & 1183.5 & $b=6.59$ & 3.14 & 12.64 \\
\hline & $\mathrm{c}=15.48$ & $\gamma=66.6$ & & $\mathrm{c}=7.74$ & & \\
\hline
\end{tabular}




$\begin{array}{cccc}\mathrm{a} & =14.0 & & \\ \exp ^{\mathrm{a},[28]} \mathrm{b}=6.8 & 3.4 & 14.0 \\ \mathrm{c} & =7.8 & & \end{array}$

${ }^{\mathrm{a}}$ Tosylate-doped PEDOT.
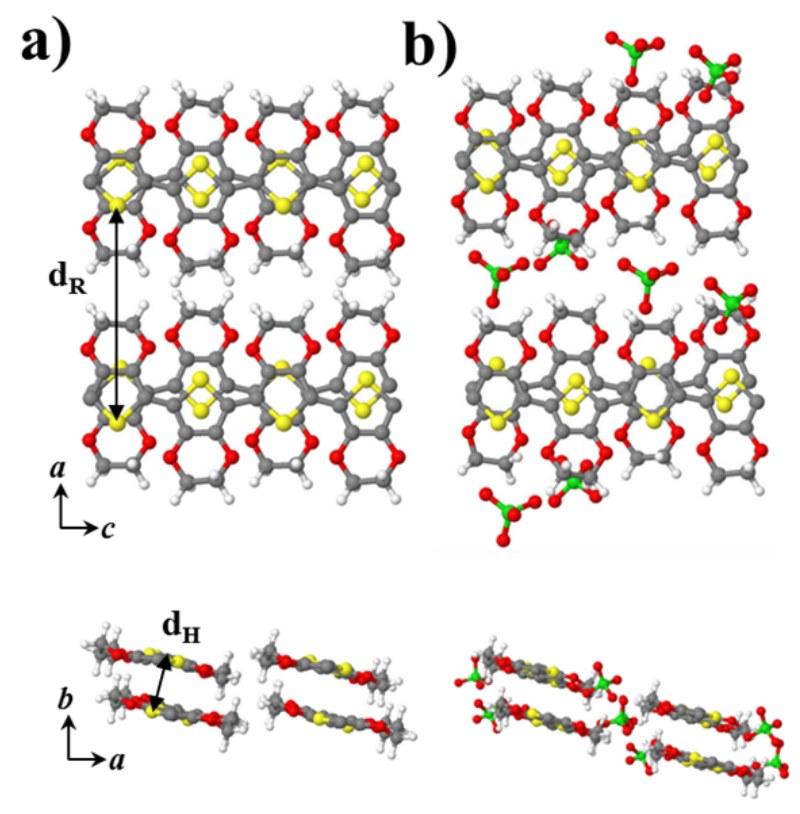

Fig. 2. Top and Side Views of the Optimized (a) Dedoped and (b) Doped PEDOT Bulk Models as Derived from 3D-PBC DFT Calculations. $d_{H}$ and $d_{R}$ chain distance are also shown.

From the theoretical and experimental parameters reported in Table 1, comparison of PEDOT's dedoped and doped structures reflects a compactness in the stacking distance of $0.3 \AA$, but also a significant increase in PEDOT inter-chains distances of $2.36 \AA$. The latter is clearly due to the disposition of the dopant perchlorate anions. Moreover, it is also observed a small elongation of $0.16 \AA$ along the axis of growth on the doped polymer. 
Dedoped and doped surface structures. Optimized structures of two multilayers with a large buffer along the lattice vector $\boldsymbol{b}$ to model a polymer surface were obtained using the 2D-PBC DFT approach. Table 2 lists the structural parameters of dedoped PEDOT and $\mathrm{ClO}_{4}{ }^{-}$doped PEDOT surface systems (hereafter $s$-dedoped and $s$-doped, respectively). An expected small contraction, which is due to the loss of periodicity in one of the crystallographic lattice direction, is observed. Furthermore, there is an important crystal restructuring with a change in the main angles of the unitary cell. Indeed, we can see how the quasi-monoclinic dedoped bulk crystal becomes almost a quasi-orthorhombic system on the calculated surface model, the $\gamma$ angle increasing from $81.9^{\circ}$ to $86.8^{\circ}$, meanwhile the other two unit cell angles remains close to $90^{\circ}$. In contrast, in the $s$-doped system $\gamma$ increases from $66.6^{\circ}$ to $73.2^{\circ}$ while the cells maintains the triclinic geometry.

Table 2. Calculated Values on the Optimized Surface Structures of the Supercell's Vectors (Distances and Angles in $\AA$ and ${ }^{\circ}$, respectively), Volume (V; in $\left.\AA^{3}\right)$, Inter-chain Stacking Distance $\left(\mathrm{d}_{\mathrm{H}}\right.$; in $\AA$ ) and Parallel Interchain Distance $\left(\mathrm{d}_{\mathrm{R}}\right.$; in $\AA$ ) of Dedoped and Doped PEDOT of Raw Surface Models ( $(s-)$ and Nanopored (np-) Surface Models.

\begin{tabular}{|c|c|c|c|c|c|c|c|c|c|}
\hline \multirow[t]{2}{*}{ Surface } & \multicolumn{3}{|c|}{ Slab $^{a}$} & \multicolumn{3}{|c|}{ Angles } & \multirow[t]{2}{*}{$\mathbf{V}$} & \multirow[t]{2}{*}{$d_{H}$} & \multirow[t]{2}{*}{$\mathbf{d}_{\mathrm{R}}$} \\
\hline & $a$ & $b$ & $c$ & $\alpha$ & $\beta$ & $\gamma$ & & & \\
\hline$S$-dedoped & 20.40 & 6.48 & 15.67 & 91.1 & 89.9 & 86.8 & 2069 & 3.24 & 10.20 \\
\hline$s$-doped & 24.51 & 6.44 & 15.44 & 85.0 & 87.1 & 73.2 & 2325 & 3.22 & 12.26 \\
\hline$n p$-dedoped ${ }^{b}$ & 20.50 & 6.54 & 15.67 & 90.4 & 89.8 & 92.0 & 2096 & 3.27 & $10.18^{d}$ \\
\hline$n p$-doped ${ }^{b}$ & 23.38 & 6.54 & 15.43 & 85.6 & 87.1 & 74.6 & 2362 & 3.27 & $12.18^{d}$ \\
\hline
\end{tabular}


Overall, a reduction on the interchain distances is observed when comparing bulk and surface models, which is mainly due to the periodicity reduction. Indeed, $s$-dedoped presents a large reduction $(-5.8 \%)$ on the $d_{H}$ interchain distance (perpendicular to the surface), whereas on the other lattice direction $\left(d_{R}\right)$ the reduction on the interchain distances is slightly lower $(-2.4 \%)$. Furthermore, $s$-doped presents different figures than $s$-dedoped when is compared with the $b$ doped crystal. Specifically, the $d_{H}$ interchain distance increases about $+2.5 \%$ meanwhile $d_{R}$ is compacted on about $-3 \%$ when is compared with $b$-doped model.

The doping effect on the surface models leads to a supercell volume increase of $12.4 \%$. The slab volume was calculated following the general unit cell volume formula ( $\mathrm{V}=\boldsymbol{a} \times \boldsymbol{b} \times \boldsymbol{c}$ ) but considering the $\boldsymbol{b}$ lattice vector as the double of the interchain $\mathrm{d}_{\mathrm{H}}$ distance. This figure comes from the necessary distance increase between parallel PEDOT chains $\left(d_{R}\right)$ to accommodate the perchlorate dopant ions into such interchain space with a $\Delta \mathrm{d}_{\mathrm{R}}=2.06 \AA$, which represents an increment of $20 \%$. Similar figures were obtained when comparing $b$-dedoped and $b$-doped models with $\Delta \mathrm{d}_{\mathrm{R}}=2.19 \AA$ ( $21 \%$ increase $)$.

Nanopored surface structure. Fig. 3 shows the optimized structure of dedoped and $\mathrm{ClO}_{4}{ }^{-}$ doped nanopored polymer surface models (hereafter $n p$-dedoped and $n p$-doped, respectively), which were obtained using a 2D-PBC DFT approach. The two models are made of two nanopored multilayered systems with a large buffer along $\boldsymbol{b}$ lattice vector and a crystal defect induced by 4 EDOT units missing in both layers. In order to accommodate the defect we doubled the supercell along $c$ lattice direction (8 EDOT units) compared with the $s$-dedoped and $s$-doped surface models (4 EDOT units). Table 2 lists the structural parameters of both nanopored 
structures. The supercell dimension has been normalized to be compared with the $s$-surface models holding only 4 EDOT units along $c$ lattice vector.

As it can be seen, there are not much structural differences in the slab surface dimensions due to the presence of a nano-pore defect in the surface. It is observed a slight increment on the $\boldsymbol{b}$ lattice vector $(\sim 1 \%)$ when the $n p$-models are compared with the corresponding $s$-models and, in addition, a short contraction along $\boldsymbol{a}$ lattice vector is detected for the $n p$-doped system. Consequently, a very similar volume increment is observed for both $s$ - and $n p$-doped systems ( $12.4 \%$ and $12.7 \%$, respectively) with respect to the $s$ - and $n p$-dedoped models.

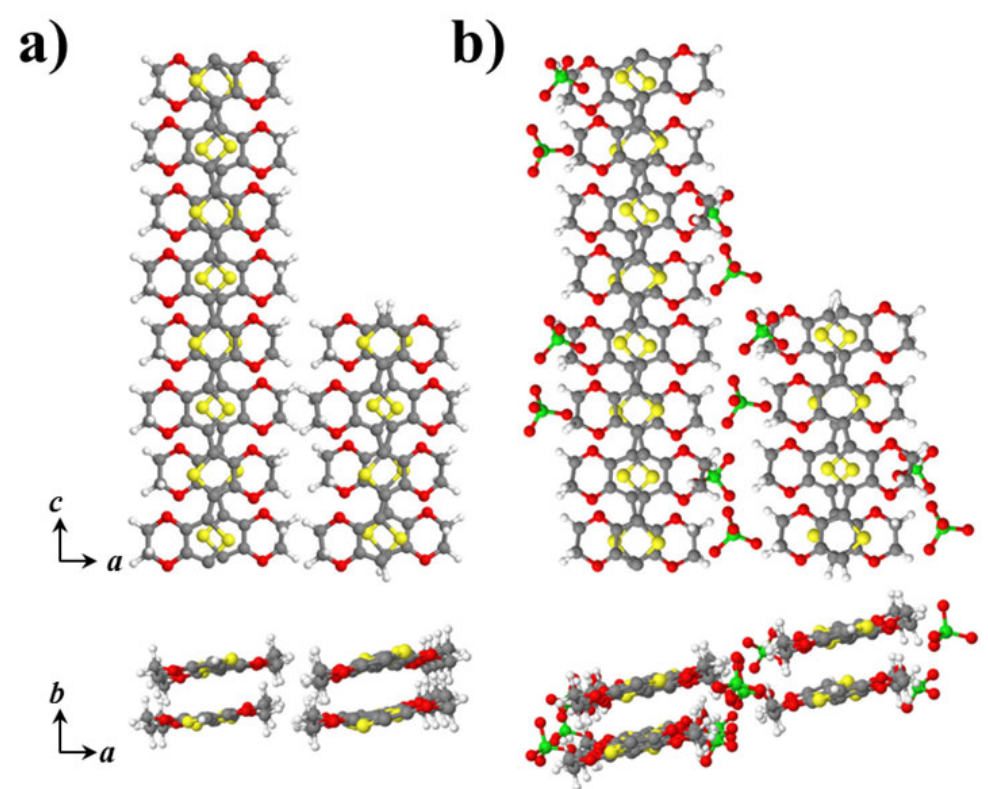

Fig. 3. Top and Side Views of the Optimized Nanopored (a) Dedoped (np-Dedoped) and (b) Doped ( $n p$-Doped) PEDOT Surface Models as Derived from 2D-PBC DFT Calculations.

On the other hand, the pore dimensions are much more affected on the surface model because of the distribution of the dopant anions. Fig. S1 (see supplementary information) shows the surface pore reconstruction in a supercell of $2 \times 2$ slabs and the pore dimension for both $n p$ - 
models. Despite of a short contraction of the pore along the $\boldsymbol{a}$ lattice vector $(\sim 1.1 \AA)$ there is a large increment of $3.3 \AA$ along $c$ lattice vector, which leads to $32.5 \%$ of the pore-surface increment after $n p$-dedoped system doping (i.e., $15.1 \%$ of circular pore-diameter increment). This large surface increment would allow to release potentially immobilized drugs inside the surface of dedoped PEDOT after doping, enabling the control on drug release by regulating the doping level.

Table 3. Averaged Inter-Ring Dihedral Angle $\left(\theta ;{ }^{\circ}\right)$, Intrachain $S \cdots S$ Distance $\left(d_{S-S}^{\text {intra }} ; \AA\right)$,

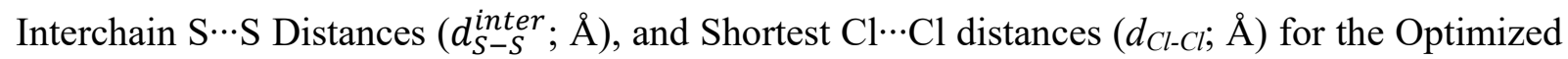
Bulk (b-), Raw Surface (s-) and Nanopored Surface (np-) Models of Dedoped and Doped PEDOT.

\begin{tabular}{cccccccc}
\hline Surface & $\boldsymbol{\theta}$ & $\boldsymbol{d}_{\boldsymbol{S}-\boldsymbol{S}}^{\text {intra }}$ & \multicolumn{2}{c}{$\boldsymbol{d}_{\boldsymbol{S}-\boldsymbol{S}}^{\text {inter }}$} & \multicolumn{3}{c}{$\boldsymbol{d}_{\boldsymbol{C l}-\boldsymbol{C l}}$} \\
\hline$b$-dedoped & $177.1 \pm 1.2$ & $4.47 \pm 0.00$ & $3.56 \pm 0.00$ & $4.78 \pm 0.00$ & - & - & - \\
& & & & & & \\
-doped & $176.7 \pm 1.4$ & $4.45 \pm 0.00$ & $3.38 \pm 0.04$ & $4.47 \pm 0.05$ & $5.21 \pm 0.00$ & $4.93 \pm 0.00$ & $5.33 \pm 0.00$ \\
& & & & & & - & - \\
$s$-dedoped & $174.2 \pm 3.3$ & $4.44 \pm 0.00$ & $3.40 \pm 0.00$ & $4.80 \pm 0.00$ & - & - & \\
$s$-doped & $175.3 \pm 3.2$ & $4.46 \pm 0.00$ & $3.38 \pm 0.00$ & $4.96 \pm 0.04$ & $5.09 \pm 0.00$ & $4.65 \pm 0.00$ & $5.38 \pm 0.00$ \\
$n p$-dedoped & $170.0 \pm 2.7$ & $4.44 \pm 0.00$ & $3.53 \pm 0.01$ & $4.70 \pm 0.06$ & - & - & - \\
$n p$-doped & $174.7 \pm 3.5$ & $4.44 \pm 0.00$ & $3.41 \pm 0.01$ & $4.89 \pm 0.01$ & $5.05 \pm 0.13$ & $4.69 \pm 0.02$ & $4.57 \pm 0.28$ \\
\hline
\end{tabular}

Structural differences among models. Table 3 compares selected structural parameters for all the calculated dedoped and doped PEDOT models. Interestingly, notable distortions from planarity are observed on the inter-ring dihedral angles $(\theta)$ of PEDOT chains, which are defined by the $\mathrm{S}-\mathrm{C}-\mathrm{C}-\mathrm{S}$ sequence. More specifically, the averaged inter-ring dihedral angle is getting 
further away from the ideal anti conformation $\left(\theta=180^{\circ}\right)$ as the model is losing periodicity (i.e. $\theta$ $=177.1^{\circ}, 174.2^{\circ}$, and $170.0^{\circ}$ for the $b-, s$ - and $n p$-dedoped models, respectively). Similar behavior is observed on doped models with averaged inter-ring dihedral angles of $\theta=176.7^{\circ}$, $175.3^{\circ}$ and $174.7^{\circ}$ for the $b$-, $s$ - and $n p$-systems, respectively.

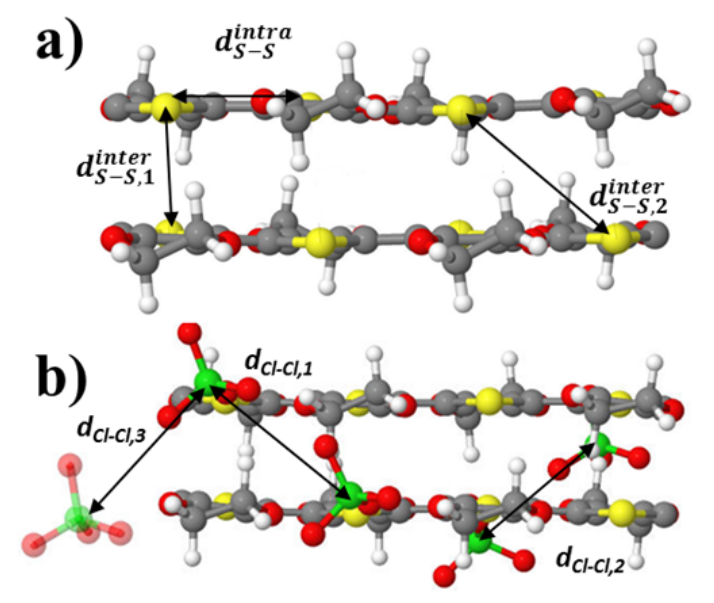

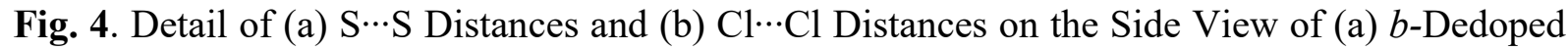
and (b) $b$-Doped Models of PEDOT. Glassy $\mathrm{ClO}_{4}{ }^{-}$Represents the Closest Virtual Image.

Fig. 4 shows intra- and intermolecular distances measured between sulphur and chlorine atoms within the supercell. Intramolecular $\mathrm{S} \cdots \mathrm{S}$ distances present close values among all optimized systems $\left(d_{S-S}^{\text {intra }}=4.45 \pm 0.05 \AA\right)$, differences being attributed to the planarity degree of the polymer chain previously discussed. On the other hand, comparison of the intermolecular $\mathrm{S} \cdots \mathrm{S}$ distances for $b$ - and $s$-dedoped models reveals a short reduction of both $d_{S-S, 1}^{\text {inter }}$ and $d_{S-S, 2}^{\text {inter }}$ (see Fig. 4). This observation is consistent with a compaction effect, which was previously discussed. Similarly, some reorganization is observed for $n p$-doped, in which the $\mathrm{S} \cdots \mathrm{S}$ distances take larger values, especially on the $d_{S-S, 2}^{\text {inter }}$ distance, due to the distortions induced by the periodicity break 
along chain axis and the subsequent reorganization of the repetitive units closest to the crystallographic defect. Nevertheless, the obtained inter-chain $\mathrm{S} \cdots \mathrm{S}$ distances are significantly lower than those previously reported from 1D-PBC DFT calculations on PEDOT doped with $\mathrm{ClO}_{4}{ }^{-}$ions $\left(\mathrm{d}_{\mathrm{S}-\mathrm{S}}^{\mathrm{inter}}=5.89 \AA\right)$. [30] Thus, the present study indicates that compaction effect is larger than reported in previous works.

Table 3 lists the three closest $\mathrm{Cl} \cdots \mathrm{Cl}$ distances among pairs of $\mathrm{ClO}_{4}^{-}$ions for the doped PEDOT models, while Fig. 4 shows their location within unit cell. It is observed that compaction effect on parallel chains also leads to the reorganization of the dopant ions, which is reflected by differences in their $\mathrm{Cl} \cdots \mathrm{Cl}$ distances. Specifically, $\mathrm{d}_{\mathrm{Cl}-\mathrm{Cl}, 1}$ and $\mathrm{d}_{\mathrm{Cl}-\mathrm{Cl}, 2}$ (see Fig. 4) present some reduction when the periodicity of the surface is lost. However, the distance involving one virtual dopant ion $\left(\mathrm{d}_{\mathrm{Cl}-\mathrm{Cl}, 3)}\right)$, which corresponds to the closest periodic image, presents a major reorganization. This has been attributed to the pore surface defect, with a reduction of about 0.76 $\AA$ when is compared with the $b$-doped model.

Table 4. HOMA Aromaticity Index for All Dedoped and Doped PEDOT systems.

\begin{tabular}{ccccccc}
\hline System & $\boldsymbol{b}$-dedoped & $\boldsymbol{b}$-doped & $\boldsymbol{s}$-dedoped & $\boldsymbol{s}$-doped & $\boldsymbol{n p}$-dedoped & $\boldsymbol{n} \boldsymbol{p}$-doped \\
\hline HOMA & 0.607 & 0.550 & 0.696 & 0.558 & 0.711 & 0.576 \\
$\mathrm{HOMA}_{\mathrm{c}}{ }^{a}$ & - & - & - & - & 0.710 & 0.573 \\
$\mathrm{HOMA}_{\mathrm{p}}{ }^{b}$ & - & - & - & - & 0.713 & 0.588
\end{tabular}

${ }^{a}$ PEDOT chains without any crystallographic defect; ${ }^{b}$ PEDOT chains that have been shortened because of the surface pore modelling.

Table 4 lists the averaged Harmonic Oscillator Model of Aromaticity (HOMA index) for all systems under study. HOMA[46] index allows to quickly and easily assign the aromatic character of any system based on the $\mathrm{C}-\mathrm{C}$ and $\mathrm{C}-\mathrm{S}$ bond length alternation pattern along the $\pi$ - 
system. This methodology has been previously used to study the aromaticity of different thiophene based polymers.[47] Within this context, the HOMA index is equal to zero for a Kekulé structure formed by a typical aromatic system with single and double bonds arranged alternatively, whereas it is equal to 1 for systems with all bonds equal to the optimal aromatic values. The inspection of the HOMA indexes shows an increase on the aromaticity values as the models loss the periodicity, and less interaction with their virtual images is observed. Also, a general loss of aromaticity is shown on all doped models when they are compared with the corresponding dedoped models.

It is known that dopant molecules induce important changes on the single and double bond alternation pattern favoring the Kekulé structure, exhibiting a transition from aromatic to quinoid-like structure, and thus, reducing the HOMA aromaticity index.[29, 36] Similarly, the HOMA index is affected by the pore defect when the aromaticity index of the two different PEDOT chains contained in the $n p$-models, are compared. These correspond to the indexes calculated considering independently the chains that are next to the pore (HOMAp in Table 4) and the chains that are out of the pore $\left(\mathrm{HOMA}_{\mathrm{c}}\right.$ in Table 4). Although it is observed a minimum loss of aromaticity for the $n p$-dedoped model, this effect becomes more pronounced for the $n p$ doped model with an increase of $2.6 \%$ on the HOMA value.

Surface morphological study. Theoretical calculations discussed in previous sub-sections show important changes in the volume and in the surface area of the nanopores when dedoped (reduced) PEDOT coverts into doped (oxidized). In order to measure experimentally the variation on the porous size with the doping level (i.e. oxidation state) a morphological comparison between PEDOT $/ \mathrm{p}^{\text {red }}$ and PEDOT $/ \mathrm{p}^{\text {oxd }}$ films was conducted. Doping level values $(d l)$ 
were obtained by $\mathrm{CV}$ on both PEDOT $/ \mathrm{p}^{\text {red }}$ and PEDOT $/ \mathrm{p}^{\text {oxd }}$ films. PEDOT $/ \mathrm{p}^{\text {oxd }}$ film presents a $d l$ value of 0.42 , close to those observed in most of the polythiophene derivatives, [27] whereas the reduced film shows a $d l$ value of 0.06 , low enough to be helpful on the pore size comparison among both polymers.

In order to obtain the two films at different $d l$, several steps had to be performed. Initially, a PEDOT sample was obtained by anodic polymerization. Fig. 5a, which displays a representative SEM micrograph of such pristine PEDOT film, shows small aggregates connected by dense networks of thin fiber-like structures. Thus, the as prepared PEDOT surface presents a porous morphology, facilitating the dopant ions movement during oxidation and reduction processes. In order to facilitate the measurement of the pore size, the surface porosity was enhanced by immersing the pristine PEDOT film in a $\mathrm{NaCl}$ aqueous solution (Fig. 1b), which resulted in the formation micrometric salt crystals on the film surface. Fig. $5 \mathrm{~b}$ shows a representative SEM micrograph of the resulting PEDOT/ $\mathrm{NaCl}$ film. As it can be seen, $\mathrm{NaCl}$ crystals with a regular and parallelepiped-shape grew among already existing conducting polymer clusters, enhancing the size of the surface cavities. Indeed, the porosity enhanced by this methodology only affects the film surface. Further characterization of $\mathrm{PEDOT} / \mathrm{NaCl}$ films is provided in Fig. S2, which shows SEM micrographs and EDX spectra of two specific surface film points that allow perfectly differentiating between the salt crystals and the polymer matrix. Although the PEDOT surface initially acts as a template, the crystal growing process causes structural distortions. Microcrystals were rinsed from the surface with water whilst their induced cavity remains (Fig. 1b), resulting in a bolstered porous PEDOT/p film. Thus, pores from pristine PEDOT converts into a set of larger pores once the $\mathrm{NaCl}$ crystals are removed from the surface. The surface porosity enhancement will facilitate doping and dedoping processes. The new pores obtained 
from rinsed $\mathrm{NaCl}$ crystals are located on the surface of PEDOT film with an observed size on the macropore length-scale (Fig. 5). Since this process is previous to doping level adjustment of each sample, initial film macroporosity won't interfere on the experimental measurement of the final nanopore size that will be done at a lower length-scale.
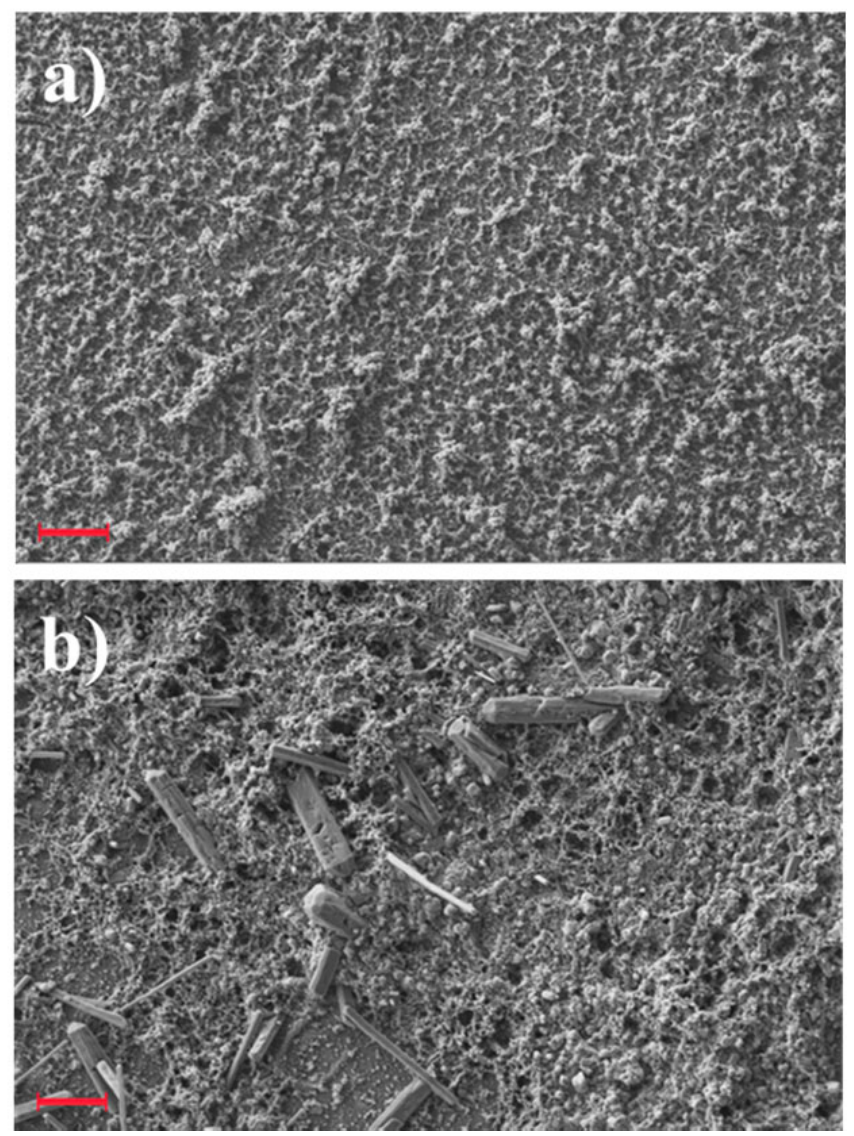

Fig. 5. Representative SEM micrographs of (a) pristine PEDOT and (b) PEDOT/NaCl films (scale bar: $10 \mu \mathrm{m}$ ).

The enhanced porous PEDOT/p film was reduced and oxidized by chronoamperometry at a constant potential of -1.1 and $1.1 \mathrm{~V}$, respectively, to obtain the PEDOT/p $\mathrm{p}^{\mathrm{red}}(d l=0.06)$ and PEDOT $/ \mathrm{p}^{\text {oxd }}(d l=0.42)$ films. Representative SEM micrographs of PEDOT $/ \mathrm{p}^{\text {red }}$ and PEDOT $/ \mathrm{p}^{\text {oxd }}$ films with a magnification of 200,000 are displayed in Figs. 6a, and 6b, respectively. Clusters of 
similar globular structures are observed in both films, even though the pore size is apparently larger for PEDOT $/ \mathrm{p}^{\text {oxd }}$ than for PEDOT $/ \mathrm{p}^{\text {red }}$. The enhanced porosity of the precursor PEDOT $/ \mathrm{p}$ film allows faster movements for the dopant ions during the both reduction and oxidation steps. The scape of the $\mathrm{ClO}_{4}{ }^{-}$ions from the PEDOT matrix in the reduction process leads to a more compact porous structure than its oxidized form, in which the dopant ions enter into the polymer matrix. The thicknesses and roughness of PEDOT $/ \mathrm{p}^{\text {red }}$ and PEDOT $/ \mathrm{p}^{\text {oxd }}$ has been determined by contact profilometry. The thicknesses of dedoped and doped films are $l \approx 4.8$ and $l \approx 6.3 \mu \mathrm{m}$, respectively, whereas the roughnesses are $R_{a} \approx 1.0$ and $R_{a} \approx 2.1 \mu \mathrm{m}$, respectively. It is observed a similar film thickness in both films, although we can still see a different roughness that might show a higher micro-porosity on doped film.
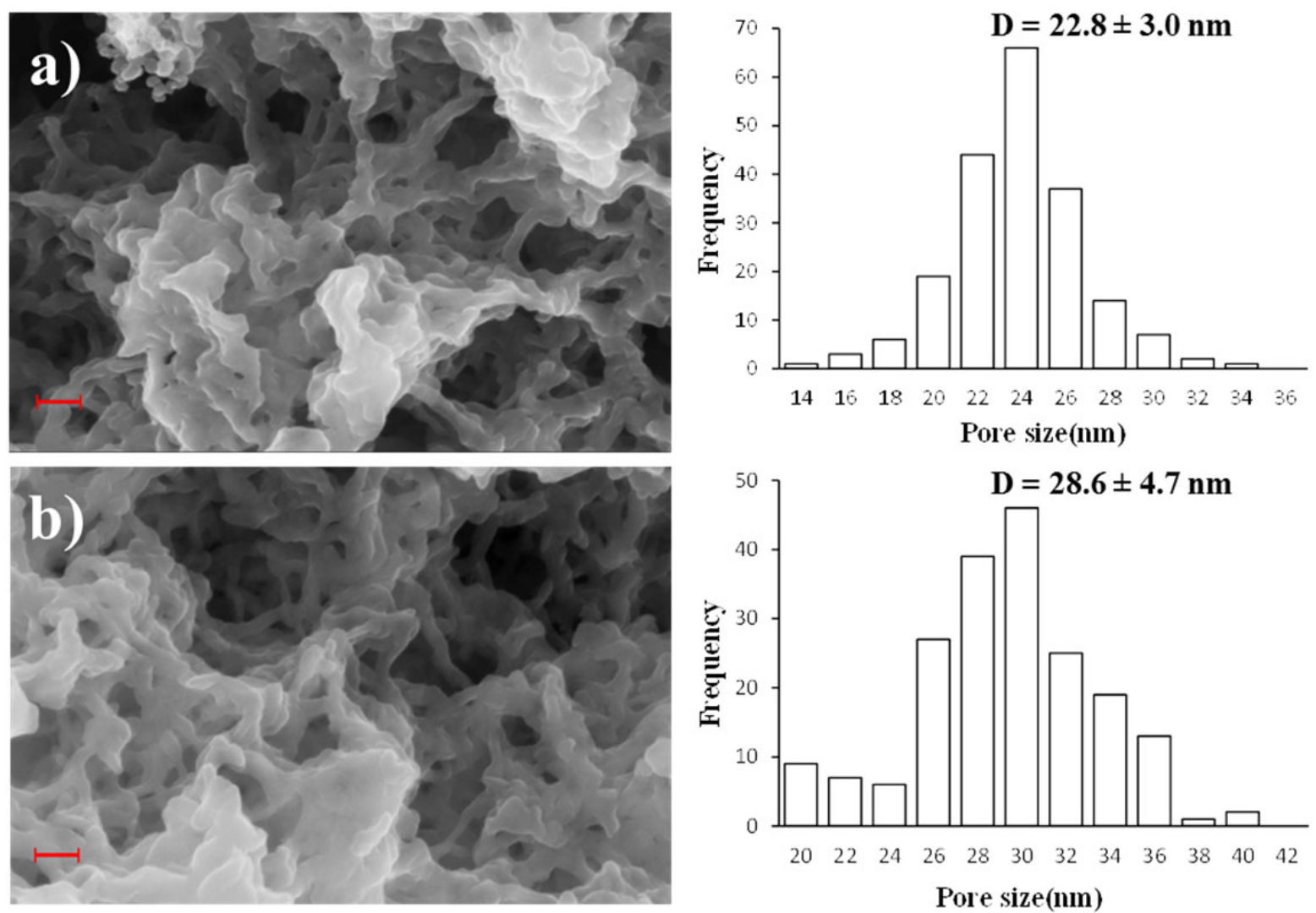
Fig. 6. Representative SEM micrographs (left, magnification: 200k, scale bar: $100 \mathrm{~nm}$ ) and nanopores size histogram derived from SEM measurements (right) of (a) PEDOT $/ \mathrm{p}^{\text {red }}$ and (b) $\mathrm{PEDOT} / \mathrm{p}^{\text {oxd }}$ films.

FTIR spectra of both PEDOT $/ \mathrm{p}^{\text {oxd }}$ and PEDOT $/ \mathrm{p}^{\text {red }}$ are shown in Fig. S3a (supplementary information). The comparative analysis presents an IR band at $985 \mathrm{~cm}^{-1}$, which is assigned to C$\mathrm{S}$ bond vibration [48], and the $1420 \mathrm{~cm}^{-1}$ band that is assigned to the symmetric stretching of the $\mathrm{C}=\mathrm{C}$ bond. Within the oxyethylene ring the IR bands at approximately 1087 and $1215 \mathrm{~cm}^{-1}$ corresponds to the $\mathrm{C}-\mathrm{O}-\mathrm{C}$ bond stretching $[48,49]$, meanwhile the band at $1369 \mathrm{~cm}^{-1}$ is assigned to the $\mathrm{CH}_{2}$ bending [44]. Furthermore, Raman spectra were taken from both PEDOT/p ${ }^{\text {oxd }}$ and PEDOT/p ${ }^{\text {red }}$ films (Fig. S3b). The Raman bands located at approximately 576, 698, 857, 991, and $1561 \mathrm{~cm}^{-1}$ are assigned to the $\mathrm{C}-\mathrm{O}-\mathrm{C}, \mathrm{C}-\mathrm{S}-\mathrm{C}, \mathrm{O}-\mathrm{C}-\mathrm{C}$, oxyethylene ring deformations and $\mathrm{CH}_{2}$ bending, respectively. Moreover, the band at 1368 and $1434 \mathrm{~cm}^{-1}$ are assigned to the $\mathrm{C}_{\alpha}-\mathrm{C}_{\beta}$ stretching and the $\mathrm{C}_{\alpha}=\mathrm{C}_{\beta}$ symmetrical bond stretching of the thiophene ring, respectively [50]. Interestingly, a red shift of about 20 and $4 \mathrm{~cm}^{-1}$ on the $\mathrm{C}_{\alpha}-\mathrm{C}_{\alpha}$ inter-ring stretching $\left(1271 \mathrm{~cm}^{-1}\right)$ and $\mathrm{C}_{\alpha}=\mathrm{C}_{\beta}$ antisymmetric stretching $\left(1517 \mathrm{~cm}^{-1}\right)$ of PEDOT $/ \mathrm{p}^{\text {red }}$ are observed, when it is doped. This is due to the PEDOT ability to change from aromatic-like structure to quinoid-like structure when doped with $\mathrm{ClO}_{4}^{-}$. The $\pi$-electrons becomes more delocalized in the quinoid-like structure due to the increase of planarity between two consecutive PEDOT rings, and thus with an increase of the system conductivity [49].

X-ray diffraction (XRD) patterns of PEDOT powder at different doping levels are shown in Fig. 7. The diffraction peak at scattering angles $(2 \theta)$ of $6.20^{\circ}$ and $6.14^{\circ}$ can be ascribed to the (100) planes of the PEDOT $/ \mathrm{p}^{\text {red }}$ and PEDOT $/ \mathrm{p}^{\text {oxd }}$, respectively [28]. Thus, the parallel interchain distances (along $\boldsymbol{a}$ crystal axis, $\boldsymbol{d}_{\boldsymbol{R}}$ ) of both samples are very close with values of 14.2 and $14.4 \AA$, 
respectively. Comparing with the value of $12.64 \AA$ derived from the theoretical model used in this work (Table 1) it appears to underestimate the theoretical $\boldsymbol{d}_{\boldsymbol{R}}$ distances in $\sim 10 \%$, which may lead to a lower threshold on the nanoporous size estimation. The diffraction hump centered about $2 \theta \approx 12^{\circ}$ corresponds to the $\mathrm{Si}$ wafer and the vacuum grease used mounting the XRD samples. From the diffraction patterns, it is observed that both dedoped and doped samples presents a very low crystallinity of $\sim 5 \%$ that can be evaluated by the relationship between the crystalline peaks area and the total area under the diffraction pattern [51].

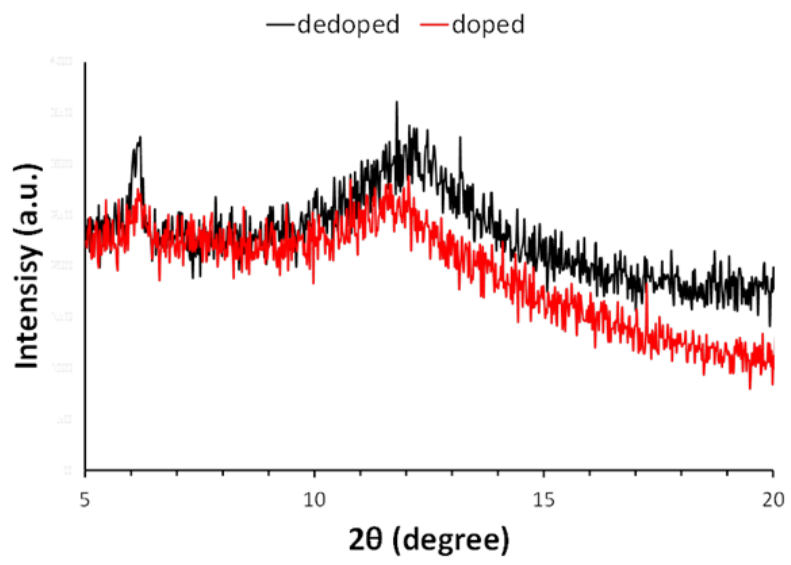

Fig. 7. XRD patterns of PEDOT $/ \mathrm{p}^{\text {red }}$ (dedoped, black line) and PEDOT $/ \mathrm{p}^{\text {oxd }}$ (doped, red line) films.

The density of PEDOT $/ \mathrm{p}^{\text {red }}$ and PEDOT $/ \mathrm{p}^{\text {oxd }}$ has been obtained by means of the flotation method with values of 1.69 and $1.73 \mathrm{~g} / \mathrm{cm}^{3}$, respectively. The PEDOT/ $\mathrm{p}^{\text {red }}$ density is close to the theoretical density calculated by the $b$-dedoped crystal $\left(\mathrm{d}=1.57 \mathrm{~g} / \mathrm{cm}^{3}\right)$, however, the PEDOT $/ \mathrm{p}^{\text {oxd }}$ system present a much lower density than that on the $b$-doped theoretical crystal $\left(\mathrm{d}=2.13 \mathrm{~g} / \mathrm{cm}^{3}\right)$. Still, before a direct comparison among theoretical and experimental density values some considerations have to be taken. Synthetized reduced and oxidized PEDOT films are semicrystalline polymers with a low degree of crystallinity ( $\sim 5 \%$, see above), and consequently, with a higher percentage of amorphous domains. The density of the crystalline domain is 
typically larger than that on the amorphous domain since the polymer chains are more tightly packed than the amorphous region [52]. Thus, the measured density of PEDOT will be expected to be an intermediate value between both domains. On the other hand, synthetized films present some different doping level that is not equal to the idealized theoretical system. So, after correcting the amount of dopant ions $(\mathrm{dl}=0.06)$ the density of $b$-dedoped system is $1.64 \mathrm{~g} / \mathrm{cm}^{3}$, in good agreement with the experimental value. However, the high density value obtained by the $b$ doped system can be explained not only by its doping level difference with the synthetized film $(\mathrm{dl}=0.42$ instead of 0.50$)$ but for the $10 \%$ of error in the length of parameter $\boldsymbol{a}$ on the crystal unit cell (see above). After correcting both points a theoretical density of $\sim 1.80 \mathrm{~g} / \mathrm{cm}^{3}$ is obtained, which is close to the experimental value.

Three different samples of PEDOT $/ \mathrm{p}^{\text {red }}$ and PEDOT $/ \mathrm{p}^{\text {oxd }}$ were used to measure the average pore size, which was $22.8 \pm 3.0$ and $28.6 \pm 4.7 \mathrm{~nm}$, respectively. Although both theoretical and experimental values points qualitatively to an increment of the pore surface upon oxidation, there is a clear quantitative difference. Thus, the increment of the pore size observed experimentally is $25.2 \%(56.9 \%$ of pore-surface increment $)$ while that derived from theoretical calculations is $15.1 \%(32.5 \%$ of pore-surface increment). Obviously, this difference has been attributed to the different length-scales of theoretical calculations $(\sim 1.5 \mathrm{~nm})$ and experimental measures $(\sim 30.0$ $\mathrm{nm}$ ) but also due to the $\sim 10 \%$ of error evaluating $\boldsymbol{d}_{\boldsymbol{R}}$ of $n p$-doped system, which after correction will lead to close values ( $\sim 21 \%$ of pore size increment) to those experimentally observed.

Material properties may change dramatically when are reduced to nanoscale dimensions. In fact, from roughly about $100 \mathrm{~nm}$ and below, materials break a size barrier below which the quantum effects and electronic energy becomes relevant. Thus, materials on nanoscale can show very different properties compared to what they show on a macroscale. In this work, 
experimental pores were found and measured on the nanoscale, where the dimension of the nanofibers is similar to the observed nanopores (Fig. 6) and below $100 \mathrm{~nm}$. Thus, it is expected that quantum effects will have a noticeable importance on the material organization and nanopores formation among the nanochains of PEDOT. Even though there is a factor of $\sim 20$ between the theoretical simulated nanopores size and its experimental measurement, theoretical model (at quantum level of calculation) used to study the PEDOT surface chains organization due to the presence of a nano-void becomes a good model to capture all the quantum interactions. However, important phenomena happening during the oxidation-reduction steps, such as the surface-clustering of EDOT fibers, changes in the crystallographic system of the surface, and the ion diffusion in and out from the EDOT-matrix, might influence on the magnification of the porosity observed when the oxidized and reduced surface films are compared at the nanometric scale with the simulated values.

\section{Conclusions}

The influence of the oxidation state on the pores size has been studied in PEDOT films using theoretical calculations and experimental measures. $b$-, $s$ - and $n p$-dedoped and doped models have been simulated under periodical boundary conditions at the DFT level. The quasimonoclicic structure obtained for $b$-dedoped PEDOT evolves towards a triclinic system after doping with $\mathrm{ClO}_{4}^{-}$anions. Although the $s$-models of PEDOT keep structures similar to those $b$ models, the volume of $s$-doped is $12.5 \%$ higher than that of $s$-dedoped. Besides, $n p$-models maintain structural similarities with $s$-models, the incorporation of $\mathrm{ClO}_{4}{ }^{-}$dopant anions causing important changes in the supercell dimensions. Moreover, the size of the pore created as a structural defect in the $n p$-dedoped model increases by $15.1 \%$ in the $n p$-doped one $(\sim 21 \%$ after a 
cell parameter correction). The structural tendencies predicted by theoretical calculations have been confirmed by experimental observation. More specifically, the average pore size of PEDOT porous films has been compared after chronoamperometric reduction and oxidation. The averaged pore size of PEDOT $/ \mathrm{p}^{\text {oxd }}$ is $25.2 \%$ larger than that of PEDOT $/ \mathrm{p}^{\text {red }}$. This important change on the surface porosity of the PEDOT matrix under different oxidation states allows foreseeing important potential applications on the load and release of drugs controlled by the oxidation state of the polymeric matrix.

\section{ACKNOWLEDGMENT}

This work was supported by MINECO-FEDER (MAT2015-69367-R and RTI2018-098951-BI00 and FPI grand BES-2016-077664 to G.R.) and the Agència de Gestió d'Ajuts Universitaris i de Recerca (2017SGR359). The authors would like to acknowledge RES to give access to Mare Nostrum at Barcelona Supercomputer Centre. Support for the research of C.A. was received through the prize "ICREA Academia" for excellence in research funded by the Generalitat de Catalunya. 


\section{REFERENCES}

[1] T.A. Desai, D.J. Hansford, L. Kulinsky, A.H. Nashat, G. Rasi, J. Tu, Y. Wang, M. Zhang, M. Ferrari, Nanopore Technology for Biomedical Applications, Biomedical Microdevices 2(1) (1999) 11-40.

[2] X. Hou, Smart Gating Multi-Scale Pore/Channel-Based Membranes, Adv. Mater. 28(33) (2016) 7049-7064.

[3] H. Zhang, Y. Tian, L. Jiang, Fundamental studies and practical applications of bio-inspired smart solid-state nanopores and nanochannels, Nano Today 11(1) (2016) 61-81.

[4] A. Puiggalí-Jou, M.M. Pérez-Madrigal, L.J. del Valle, E. Armelin, M.T. Casas, C. Michaux, E.A. Perpète, F. Estrany, C. Alemán, Confinement of a $\beta$-barrel protein in nanoperforated freestanding nanomembranes for ion transport, Nanoscale 8(38) (2016) 16922-16935.

[5] M.M. Perez-Madrigal, E. Armelin, J. Puiggali, C. Aleman, Insulating and semiconducting polymeric free-standing nanomembranes with biomedical applications, Journal of Materials Chemistry B 3(29) (2015) 5904-5932.

[6] T. Komachi, H. Sumiyoshi, Y. Inagaki, S. Takeoka, Y. Nagase, Y. Okamura, Adhesive and robust multilayered poly(lactic acid) nanosheets for hemostatic dressing in liver injury model, Journal of Biomedical Materials Research Part B: Applied Biomaterials 105(7) (2017) 17471757.

[7] S.M. Park, H. Kim, K.H. Song, S. Eom, H. Park, J. Doh, D.S. Kim, Ultra-thin, aligned, freestanding nanofiber membranes to recapitulate multi-layered blood vessel/tissue interface for leukocyte infiltration study, Biomaterials 169 (2018) 22-34.

[8] G.G. Wallace, M.J. Higgins, S.E. Moulton, C. Wang, Nanobionics: the impact of nanotechnology on implantable medical bionic devices, Nanoscale 4(15) (2012) 4327-4347. 
[9] B. Guo, P.X. Ma, Conducting Polymers for Tissue Engineering, Biomacromolecules 19(6) (2018) 1764-1782.

[10] Kenry, B. Liu, Recent Advances in Biodegradable Conducting Polymers and Their Biomedical Applications, Biomacromolecules 19(6) (2018) 1783-1803.

[11] F.-H. Lu, M.G. Mohamed, T.-F. Liu, C.-G. Chao, S.-W. Kuo, A facile cosolvent/chelation method for the preparation of semi-crystalline $\mathrm{CuCl} 2$ (ethylene glycol)/poly(3-hexylthiophene) complexes displaying specific luminescence properties, RSC Advances 5(106) (2015) 8772387729.

[12] Y.-Z. Long, M.-M. Li, C. Gu, M. Wan, J.-L. Duvail, Z. Liu, Z. Fan, Recent advances in synthesis, physical properties and applications of conducting polymer nanotubes and nanofibers, Prog. Polym. Sci. 36(10) (2011) 1415-1442.

[13] F.-H. Lu, F.-C. Chang, M.-G. Mohamed, T.-F. Liu, C.-G. Chao, S.-W. Kuo, Conducting $\mathrm{Ag} /$ oligothiophene complex pastes through a simple quenching/chelation method, Journal of Materials Chemistry C 2(30) (2014) 6111-6118.

[14] R. Balint, N.J. Cassidy, S.H. Cartmell, Conductive polymers: Towards a smart biomaterial for tissue engineering, Acta Biomaterialia 10(6) (2014) 2341-2353.

[15] M.G. Mohamed, C.-C. Cheng, Y.-C. Lin, C.-W. Huang, F.-H. Lu, F.-C. Chang, S.-W. Kuo, Synthesis and self-assembly of water-soluble polythiophene-graft-poly(ethylene oxide) copolymers, RSC Advances 4(42) (2014) 21830-21839.

[16] J. Casanovas, D. Zanuy, C. Alemán, Conducting Polymer Actuator Mechanism Based on the Conformational Flexibility of Calix[4]arene13, Angew. Chem. Int. Edit 45(7) (2006) 1103 1105. 
[17] D. Zanuy, C. Alemán, Contraction Process of an Electroactive Actuator Based on a One Microsecond Atomistic Molecular Dynamics Simulation, Chem. - Eur. J. 13(9) (2007) 26952700.

[18] L. Valero, J. Arias-Pardilla, J. Cauich-Rodríguez, M.A. Smit, T.F. Otero, Characterization of the movement of polypyrrole-dodecylbenzenesulfonate-perchlorate/tape artificial muscles. Faradaic control of reactive artificial molecular motors and muscles, Electrochim. Acta 56(10) (2011) 3721-3726.

[19] M. Beregoi, A. Evanghelidis, V.C. Diculescu, H. Iovu, I. Enculescu, Polypyrrole Actuator Based on Electrospun Microribbons, ACS Applied Materials \& Interfaces 9(43) (2017) 3806838075.

[20] E.W.H. Jager, E. Smela, O. Inganäs, Microfabricating Conjugated Polymer Actuators, Science 290(5496) (2000) 1540-1545.

[21] M. Fuchiwaki, J.G. Martinez, T.F. Otero, Polypyrrole Asymmetric Bilayer Artificial Muscle: Driven Reactions, Cooperative Actuation, and Osmotic Effects, Adv. Funct. Mater. 25(10) (2015) 1535-1541.

[22] M.M. Pérez-Madrigal, M.I. Giannotti, L.J. del Valle, L. Franco, E. Armelin, J. Puiggalí, F. Sanz, C. Alemán, Thermoplastic Polyurethane:Polythiophene Nanomembranes for Biomedical and Biotechnological Applications, ACS Applied Materials \& Interfaces 6(12) (2014) 97199732.

[23] A. Puiggalí-Jou, J. Medina, L.J. del Valle, C. Alemán, Nanoperforations in poly(lactic acid) free-standing nanomembranes to promote interactions with cell filopodia, Eur. Polym. J. 75 (2016) 552-564. 
[24] T.F. Otero, J.G. Martinez, J. Arias-Pardilla, Biomimetic electrochemistry from conducting polymers. A review: Artificial muscles, smart membranes, smart drug delivery and computer/neuron interfaces, Electrochim. Acta 84 (2012) 112-128.

[25] T.F. Otero, Coulovoltammetric and Dynamovoltammetric Responses from Conducting Polymers and Bilayer Muscles as Tools to Identify Reaction-driven Structural Changes. A review, Electrochim. Acta 212 (2016) 440-457.

[26] A. Puiggalí-Jou, A. Cejudo, L.J. del Valle, C. Alemán, Smart Drug Delivery from Electrospun Fibers through Electroresponsive Polymeric Nanoparticles, ACS Applied Bio Materials 1(5) (2018) 1594-1605.

[27] J. Roncali, Conjugated poly(thiophenes): synthesis, functionalization, and applications, Chem. Rev. 92(4) (1992) 711-738.

[28] K.E. Aasmundtveit, E.J. Samuelsen, L.A.A. Pettersson, O. Inganäs, T. Johansson, R. Feidenhans'1, Structure of thin films of poly(3,4-ethylenedioxythiophene), Synth. Met. 101(1) (1999) 561-564.

[29] E.-G. Kim, J.-L. Brédas, Electronic Evolution of Poly(3,4-ethylenedioxythiophene) (PEDOT): From the Isolated Chain to the Pristine and Heavily Doped Crystals, J. Am. Chem. Soc. 130(50) (2008) 16880-16889.

[30] J. Casanovas, D. Zanuy, C. Aleman, Distribution of dopant ions around poly(3,4ethylenedioxythiophene) chains: a theoretical study, Phys. Chem. Chem. Phys. 19(15) (2017) 9889-9899.

[31] F. Bartha, I.A. Howard, P. Geerlings, C. Van Alsenoy, D. Vanderzande, T.J. Cleij, F. Bogár, Density functional crystal orbital study of cyano-substituted poly(para-phenylene-vinylene) and poly(quinoxaline-vinylene), Int. J. Quantum Chem. 106(8) (2006) 1912-1923. 
[32] B.G. Janesko, Comparing modern density functionals for conjugated polymer band structures: Screened hybrid, Minnesota, and Rung 3.5 approximations, J. Chem. Phys. 134(18) (2011) 184105.

[33] S.S. Zade, M. Bendikov, Twisting of Conjugated Oligomers and Polymers: Case Study of Oligo- and Polythiophene, Chemistry - A European Journal 13(13) (2007) 3688-3700.

[34] B.M. Wong, J.G. Cordaro, Electronic Properties of Vinylene-Linked Heterocyclic Conducting Polymers: Predictive Design and Rational Guidance from DFT Calculations, J. Phys. Chem. C 115(37) (2011) 18333-18341.

[35] T.P. Kaloni, P.K. Giesbrecht, G. Schreckenbach, M.S. Freund, Polythiophene: From Fundamental Perspectives to Applications, Chem. Mater. 29(24) (2017) 10248-10283.

[36] W. Shi, T. Zhao, J. Xi, D. Wang, Z. Shuai, Unravelling Doping Effects on PEDOT at the Molecular Level: From Geometry to Thermoelectric Transport Properties, J. Am. Chem. Soc. 137(40) (2015) 12929-12938.

[37] C. Ocampo, R. Oliver, E. Armelin, C. Alemán, F. Estrany, Electrochemical Synthesis of Poly(3,4-ethylenedioxythiophene) on Steel Electrodes: Properties and Characterization, J. Polym. Res. 13(3) (2006) 193-200.

[38] H. Randriamahazaka, V. Noël, C. Chevrot, Nucleation and growth of poly(3,4ethylenedioxythiophene) in acetonitrile on platinum under potentiostatic conditions, J. Electroanal. Chem. 472(2) (1999) 103-111.

[39] D. Sánchez-Portal, P. Ordejón, E. Artacho, J.M. Soler, Density-functional method for very large systems with LCAO basis sets, Int. J. Quantum Chem. 65(5) (1997) 453-461. 
[40] J.M. Soler, E. Artacho, J.D. Gale, A. García, J. Junquera, P. Ordejón, D. Sánchez-Portal, The SIESTA method for ab initio order- $\mathrm{N}$ materials simulation, J. Phys.: Condens. Matter 14(11) (2002) 2745.

[41] J.P. Perdew, K. Burke, M. Ernzerhof, Generalized Gradient Approximation Made Simple, Phys. Rev. Lett. 77(18) (1996) 3865-3868.

[42] N. Troullier, J.L. Martins, Efficient pseudopotentials for plane-wave calculations, Phys. Rev. B 43(3) (1991) 1993-2006.

[43] H.J. Monkhorst, J.D. Pack, Special points for Brillouin-zone integrations, Phys. Rev. B 13(12) (1976) 5188-5192.

[44] F. Tran-Van, S. Garreau, G. Louarn, G. Froyer, C. Chevrot, Fully undoped and soluble oligo(3,4-ethylenedioxythiophene)s: spectroscopic study and electrochemical characterization, J. Mater. Chem. 11(5) (2001) 1378-1382.

[45] B. Zhang, K. Wang, D. Li, X. Cui, Doping effects on the thermoelectric properties of pristine poly(3,4-ethylenedioxythiophene), RSC Advances 5(43) (2015) 33885-33891.

[46] T.M. Krygowski, Crystallographic studies of inter- and intramolecular interactions reflected in aromatic character of .pi.-electron systems, J. Chem. Inf. Comput. Sci. 33(1) (1993) 70-78.

[47] J. Torras, O. Bertran, C. Alemán, Structural and Electronic Properties of Poly(thiaheterohelicene)s, J. Phys. Chem. B 113(46) (2009) 15196-15203.

[48] Q. Zhao, R. Jamal, L. Zhang, M. Wang, T. Abdiryim, The structure and properties of PEDOT synthesized by template-free solution method, Nanoscale Research Letters 9(1) (2014) 557. 
[49] P. Chutia, A. Kumar, Charge-carrier relaxation dynamics and ac conductivity scaling of poly(3,4-ethylenedioxythiophene) nanoparticles, physica status solidi (a) 212(12) (2015) 27272737.

[50] W.W. Chiu, J. Travaš-Sejdić, R.P. Cooney, G.A. Bowmaker, Studies of dopant effects in poly(3,4-ethylenedi-oxythiophene) using Raman spectroscopy, J. Raman Spectrosc. 37(12) (2006) 1354-1361.

[51] S. Chandra, S. Annapoorni, F. Singh, R.G. Sonkawade, J.M.S. Rana, R.C. Ramola, Low temperature resistivity study of nanostructured polypyrrole films under electronic excitations, Nuclear Instruments and Methods in Physics Research Section B: Beam Interactions with Materials and Atoms 268(1) (2010) 62-66.

[52] V. Kholodovych, W.J. Welsh, Densities of Amorphous and Crystalline Polymers, in: J.E. Mark (Ed.), Physical Properties of Polymers Handbook, Springer New York, New York, NY, 2007, pp. 611-617. 